\title{
Structure functions and energy transfers in a decaying rotating turbulence experiment
}

\author{
F. Moisy, L. Agostini, and G. Tan \\ Université Paris-Sud; Université Pierre et Marie Curie; CNRS. Laboratoire FAST \\ (Fluides, Automatique et Systèmes Thermiques), Bâtiment 502, 91405 Orsay, \\ France \\ moisy at fast.u-psud.fr
}

A key feature of rotating turbulence is the anisotropic transfer of energy towards horizontal modes, normal to the rotation axis $\Omega=\Omega \hat{\mathbf{z}}$, eventually driving turbulence to a quasi-two-dimensional state $[1,2,3]$. While nonrotating homogeneous and isotropic turbulence shows direct energy cascade towards small scales, the energy may be transfered in homogeneous rotating turbulence both in scale and direction.

In isotropic turbulence, the energy transfers are classically described in the physical space using the 3 rd order structure function, $S_{3}(r)=\left\langle\delta_{r} u^{3}\right\rangle$, where $\delta_{r} u=[\mathbf{u}(\mathbf{x}+\mathbf{r})-\mathbf{u}(\mathbf{x})] \cdot \mathbf{r} / r$ is the longitudinal velocity increment. Exact results for this quantity are $S_{3}=-\frac{4}{5} \epsilon r$ in $3 \mathrm{D}$ turbulence, and $S_{3}=+\frac{3}{2} \epsilon r$ in $2 \mathrm{D}$ turbulence, with $\epsilon$ the rate of energy injected in the system. The sign of $S_{3}(r)$ is indeed related to the direction of the energy flux: positive (resp. negative) for transfers towards larger (resp. smaller) scales. To date, no exact anisotropic extension of the $4 / 5$ th law has been derived for homogeneous rotating turbulence. A recent attempt to derive a quasi-isotropic form of the 4/5 law under weak rotation has been carried out by Chakraborty and Bhattacharjee (2007) [4].

Recently, several experiments have reported the possibility of a change of sign of the 3rd order structure function for separations in the plane normal to $\boldsymbol{\Omega}[5,6,7]$. In the experiment of Baroud et al. [6], where turbulence is forced by radial jets originating from a circular array of source holes in a rotating annulus, a change of sign is observed on $S_{3}$. A change of sign of $S_{3}(r)$ has also been reported in our experiment of decaying grid-generating turbulence in a rotating tank [7]. This change of sign is found to occur at a scale $r_{0}$ which is decreasing during the decay, although this trend was not characterized in details in this work.

We present here experimental results of decaying turbulence in a rotating frame $[7,8,9]$, aiming to further investigate the influence of the background rotation on the $3 \mathrm{rd}$ order structure function. The experiment consists in a water filled tank of square section, of side $35 \mathrm{~cm}$ and height $44 \mathrm{~cm}$, rotating 

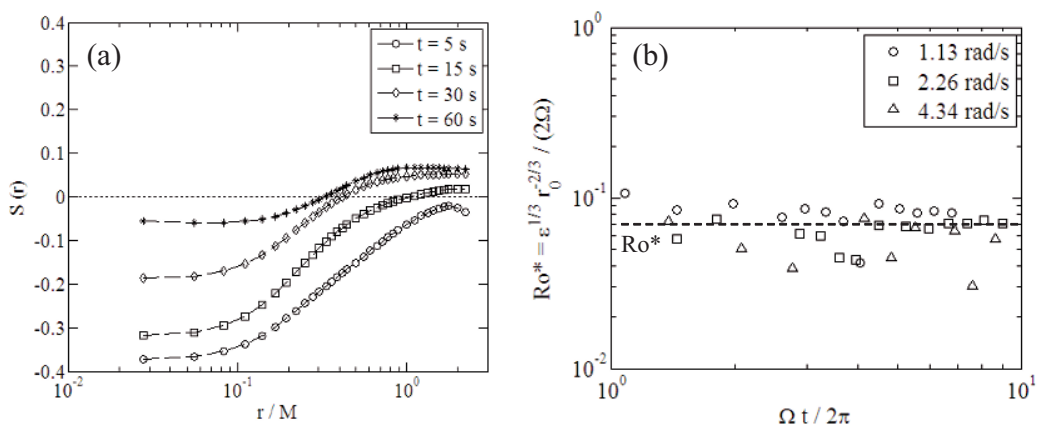

Fig. 1.1. (a) Skewness of the longitudinal velocity increments, $S(r)=$ $S_{3}(r) / S_{2}(r)^{3 / 2}$, measured in the plane normal to the rotation axis, at four times during the decay. The scale $r_{0}$ where $S_{3}$ changes sign is a decreasing function of time. (b) The local Rossby number (1.1) computed at the scale $r_{0}$, for different rotation rates.

at constant angular velocity. Turbulence is generated by towing a co-rotating square grid from the bottom to the top of the tank. Structure functions are computed from the horizontal components of the velocity in a horizontal plane at mid height of the tank, using a corotating Particle Image Velocimetry system.

Figure 1.1(a) shows that the skewness of the velocity increments, $S(r)=$ $S_{3}(r) / S_{2}(r)^{3 / 2}$, measured in a plane normal to $\Omega$, crosses zero at a scale $r_{0}$, with $r_{0}$ being a decreasing function of time. This change of sign suggests that scales $r \ll r_{0}$ transfer energy towards small scales, whereas larger scales, $r \gg$ $r_{0}$, show a behavior consistent with transfers towards larger scales. Although a positive $S_{3}$ can be clearly associated to an inverse energy cascade for forced turbulence [6], its physical signification is unclear in the case of decaying turbulence, since no energy is supplied at scale $r_{0}$.

This scale $r_{0}$ can be tentatively interpreted as the crossover scale separating $3 \mathrm{D}$ turbulence at small scales with no influence of the rotation (large Rossby number), and a flow at larger scales dominated by the rotation (low Rossby number), in analogy with the Ozmidov scale of stratified turbulence $[10,11]$. This interpretation can be indeed tested by computing a local Rossby number, defined as

$$
R o(r)=S_{2}(r)^{1 / 2} /(2 \Omega r)
$$

where $S_{2}(r)=\left\langle\delta_{r} u^{2}\right\rangle$ is the 2nd order structure function. Accordingly, $R o(r)$ should take a constant value of order unity at scale $r=r_{0}$, which we denote $R o\left(r_{0}\right)=R o^{*}$. Assuming that the 2/3 law remains verified for $r=r_{0}$, i.e. $S_{2}(r) \simeq(\epsilon r)^{2 / 3}$, one has $R o^{*}=\epsilon^{1 / 3} r_{0}^{-2 / 3} /(2 \Omega)$. This quantity, plotted in Fig. 1(b) as a function of time for various rotation rates, is indeed approximately constant, $R o^{*} \simeq 0.07 \pm 0.03$, confirming this simple picture. 

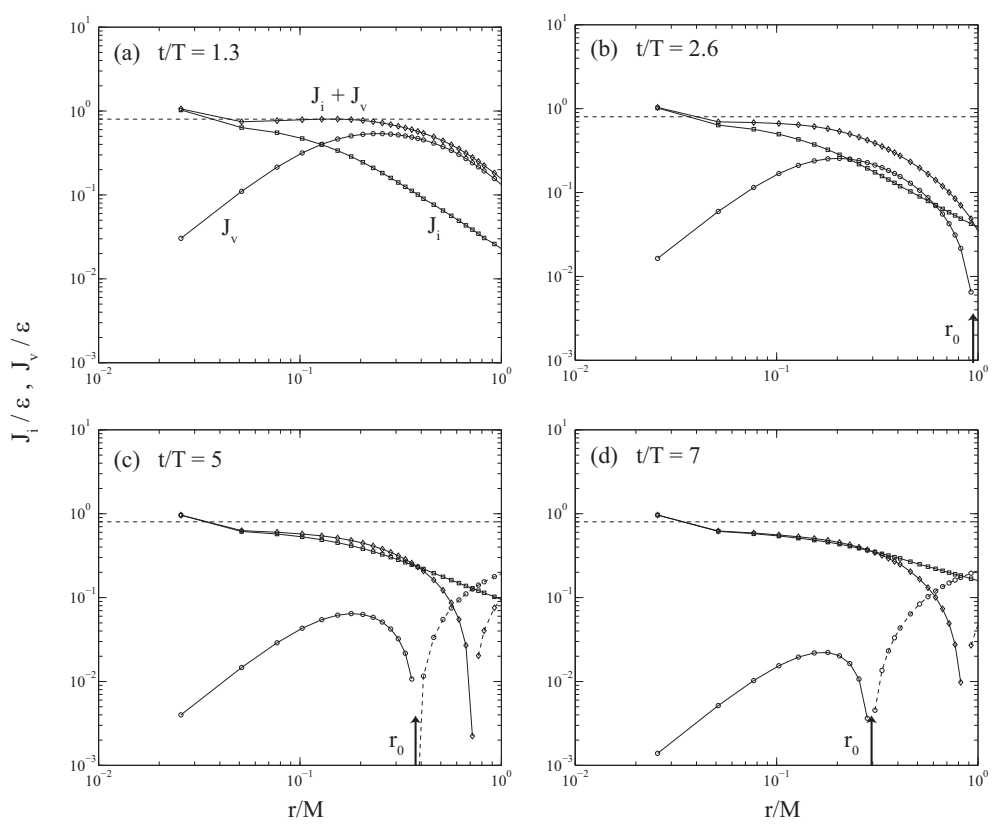

Fig. 1.2. The 2 contributions of the von Kármán-Howarth equation (1.2), $J_{i}=$ $-S_{3} / r$ and $J_{v}=6 \nu \partial S_{2} / \partial r$, and their sum $J_{i}+J_{v}$, non-dimensionalized by the instantaneous dissipation rate $\epsilon$, at 4 different times during a decay experiment with $\Omega=1.13 \mathrm{rad} \mathrm{s}^{-1}$. The horizontal dashed line shows the value $4 / 5$ expected for isotropic turbulence.

Since no accumulation of energy at large scale is observed in decaying turbulence (in particular, $S_{2}(r)$ is found to decrease in time for all $r$ ), the interpretation of a positive $S_{3}(r)$ for $r>r_{0}$ in terms of an inverse cascade is questionable. In particular, the relationship between the sign of $S_{3}$ and the direction of the energy transfers holds only in the isotropic case. For anisotropic turbulence, there is the possibility that the horizontal velocity actually cascades towards large scales, while the vertical velocity, behaving as a passive scalar advected by the horizontal motion, cascades towards small scales. Since $S_{3}(r)$ is related only to the horizontal velocity here, it may be indeed related to the direction of the energy cascade for the horizontal mode.

Another possibility is that the viscous effects may start to play a role in the energy decay at large times, for $r \simeq r_{0}$. In order to test the effect of the viscosity in the energy decay, we can measure the different contributions of the von Kármán-Howarth equation,

$$
-\frac{S_{3}}{r}+\frac{6 \nu}{r} \frac{\partial S_{2}}{\partial r}=\frac{4}{5} \epsilon,
$$

which reduces to the $4 / 5$ th law in the inertial range in the limit of large Reynolds numbers. Although this equation is not valid for anisotropic turbu- 
lence, we aim to check to what extent it remains verified at small times in decaying rotating turbulence, for measurements restricted to the horizontal plane.

The two terms of the left-hand side of Eq. (1.2), noted $J_{i}$ (inertial) and $J_{v}$ (viscous), are plotted in figure 1.2 at 4 times. At small time, the sum of the two terms remains close to the value expected for isotropic turbulence, $J_{i}+J_{v}=\frac{4}{5} \epsilon$, for all $r$. As time proceeds, when the inertial contribution $J_{i}$ crosses zero at $r=r_{0}$, the viscous contribution $J_{v}$ becomes gradually dominant, so that the sum $J_{i}+J_{v}$ remains positive at $r=r_{0}$. This would suggest that, although the nonlinear terms shows a trend towards inverse energy transfer, this effect is actually hidden by the viscous effects, resulting in an overall decay at all scales. A zero crossing of the sum $J_{i}+J_{v}$ may possibly be present at even larger scales, but this effect can not be checked by the presently available data.

More insight into the behavior of the 3rd order structure function in rotating turbulence could be gained from an isotropic generalization of the von Kármán-Howarth relation, for separations $r$ normal and perpendicular to the rotation axis. In particular, it would be of first interest to check whether the zero crossing of $S_{3}(r)$, observed both in forced [5,6] and decaying [7] turbulence, is an artifact of the restriction to horizontal velocity measurements, or a true effect that could be related to an inverse energy cascade of the $2 \mathrm{D}$ mode.

This work was supported by the ANR project no. 06-BLAN-0363-01 "HiSpeedPIV".

\section{References}

1. F. Waleffe, Phys. Fluids A 5 (3), 677 (1993).

2. C. Cambon, N. N. Mansour, and F. S. Godeferd, J. Fluid Mech. 337, 303 (1997).

3. S. Galtier, Phys. Rev. E 68, 015301R (2003).

4. S. Chakraborty, J.K. Bhattacharjee, Phys. Rev. E. 76, 036304 (2007).

5. C. Simand, F. Chillà, and J.-F. Pinton, Europhys. Lett. 49 (3), 336 (2000); 49 (6), 821 (2000)

6. C. N. Baroud, B. B. Plapp, Z.-S. She, and H. L. Swinney, Phys. Rev. Lett. 88, 114501 (2002).

7. C. Morize, F. Moisy and M. Rabaud, Phys. Fluids 17, 095105 (2005).

8. C. Morize, and F. Moisy, Phys. Fluids 18, 065107 (2006).

9. J. Seiwert, C. Morize and F. Moisy, Phys. Fluids 20, 071702 (2008).

10. O. Zeman, Phys. Fluids 6, 3221 (1994).

11. Y. Zhou, Phys. Fluids 7, 2092 (1995). 